\title{
Long non-coding RNA FOXP4-AS1 is an unfavourable prognostic factor and regulates proliferation and apoptosis in colorectal cancer
}

\author{
Juan $\mathrm{Li}^{1,2, *} \mid$ Yifan Lian ${ }^{1,2, *} \mid$ Changsheng Yan $^{3}$ | Zeling Cai ${ }^{4}$ | Jie Ding ${ }^{2}$ | \\ ${\text { Zhonghua } \mathrm{Ma}^{2} \text { | Peng Peng }}^{2}$ | Keming Wang ${ }^{1,2}$
}

\author{
${ }^{1}$ The Second Clinical Medical College of \\ Nanjing Medical University, Nanjing, Jiangsu, \\ China \\ ${ }^{2}$ Department of Oncology, Second Affiliated \\ Hospital, Nanjing Medical University, \\ Nanjing, Jiangsu, China \\ ${ }^{3}$ Department of Obstetrics and \\ Gynecology, the First Affiliated Hospital of \\ Nanjing Medical University, Nanjing, Jiangsu, \\ China \\ ${ }^{4}$ Department of General Surgery, Second \\ Affiliated Hospital, Nanjing Medical \\ University, Nanjing, Jiangsu, China \\ Correspondence \\ Keming Wang, The Second Clinical Medical \\ College of Nanjing Medical University, \\ Nanjing, China. \\ Email: kemingwang@njmu.edu.cn \\ Funding information \\ Medical Science and Technology \\ Development Foundation, Jiangsu Province \\ Department of Health, Grant/Award \\ Number: H201512; Six Talents Peak \\ Project of Jiangsu Province, Grant/Award \\ Number: WSN-050; Medical Science and \\ Technology Development Foundation of \\ Nanjing, Grant/Award Number: YKK13178; \\ Natural Science Foundation of Jiangsu \\ Province of China, Grant/Award Number: \\ BK20151578
}

\begin{abstract}
Objectives: Despite improvements in diagnosis and treatment, colorectal cancer (CRC) remains the third most common malignancy, and fourth-leading cause of cancerrelated death worldwide, and has a particularly high incidence in Western countries. Recent studies have suggested that long non-coding RNAs (IncRNAs) compose a novel class of regulators of cancer biological processes, such as proliferation, apoptosis and metastasis. Here, we report that IncRNA FOXP4-AS1 acts as a functional oncogene in CRC pathogenesis. Moreover, we have attempted to investigate the effects of FOXP4AS1 on tumour progression, both in vitro and in vivo.

Materials and methods: In this study, bioinformatic analyses and qPCR were performed to investigate FOXP4-AS1 expression in CRC tissue samples and CRC cell lines. We inhibited FOXP4-AS1 expression via FOXP4-AS1-specific siRNA transfection. Cell proliferation was assessed using cell viability and colony formation assays, as well as by flow cytometry and ethynyl deoxyuridine (Edu) analyses. Apoptosis was assessed using flow cytometry. Animal tumour xenografts were generated, and immunohistochemistry (IHC) was performed to evaluate effects of FOXP4-AS1 on CRC tumour growth in vivo. Results: We found that FOXP4-AS1 was up-regulated in CRC tissues and cell lines and that its overexpression positively correlated with advanced pathological stages and larger tumour size. Additionally, we found that FOXP4-AS1 knockdown inhibited cell proliferation and induced apoptosis. Furthermore, FOXP4-AS1 knockdown induced marked increase in number of cells in G0/G1 phase and reduction in number of cells in S phase, in DLD-1, HT-29 and HCT116 cell lines. Consistent with these findings, FOXP4-AS1 silencing inhibited tumour growth in vivo.

Conclusion: These findings suggest that FOXP4-AS1 plays a crucial role in CRC progression and may be a new biomarker in patients with CRC.
\end{abstract}

\section{1 | INTRODUCTION}

There have been dramatic improvements in the early detection and treatment of colorectal cancer (CRC); however, it remains the

${ }^{*}$ These authors contributed equally to this work. fourth-leading cause of cancer-related death in both men and women. $^{1,2}$ In 2016, an estimated 71,830 men and 65,000 women will be diagnosed with CRC, and 26,270 men and 24,040 women will die of this disease in the United States. ${ }^{1}$ Thus, it is urgent to identify novel biomarkers and therapeutic targets that are correlated with CRC tumourigenesis and progression. 
Over the past decade, human genome sequencing studies and the GENCODE project have demonstrated that only approximately $2 \%$ of the human genome comprises protein-coding genes, while the majority of the genome comprises non-coding genes encoding numerous non-coding transcripts, including microRNAs, circular RNAs and long non-coding RNAs (IncRNAs). ${ }^{3,4}$ Among these, long non-coding RNAs are emerging as crucial players in various biological processes via distinct mechanisms. ${ }^{5,6}$ They are commonly defined as transcripts greater than $200 \mathrm{nt}$ in length but have no protein-coding capacity. ${ }^{7}$ Recent experiments confirmed that IncRNA dysregulation plays a role in various malignancies. ${ }^{8-10}$ For instance, IncRNA HOTAIR overexpression facilitates breast cancer metastasis by recruiting the PRC2 complex to specific target genes genome-wide, leading to $\mathrm{H} 3 \mathrm{~K} 27$ trimethylation and epigenetic silencing of metastasis suppressor genes. ${ }^{11}$ In contrast, IncRNA maternally expressed gene 3 (MEG3) may function as a tumour suppressor partly by activating p53 in colorectal cancer. ${ }^{12}$ Therefore, a powerful suggestive link exists between IncRNAs and cancer, and IncRNAs can act as oncogenes or tumour suppressors, although the biological functions of the majority of IncRNAs remain to be elucidated.

Many studies have demonstrated that IncRNAs contribute to cancer cell phenotypes by silencing tumour suppressors or activating oncogenes. ${ }^{13,14}$ In addition, many cell cycle-related genes (including p15, p21, p27 and KLF2) act as tumour suppressors in various cancers by inhibiting the activity of kinases involved in the G1/S transition. ${ }^{15-17}$ Our previous studies have demonstrated that IncRNA BANCR (BRAF activated non-coding RNA) promotes CRC cell proliferation partly by down-regulating $\mathrm{p} 21$ expression. ${ }^{18}$

In the present study, we report for the first time that a novel IncRNA, FOXP4-AS1, was significantly up-regulated in colorectal cancer tissues. The association between FOXP4-AS1 overexpression and clinicopathological characteristics was also studied. Additionally, FOXP4-AS1 knockdown inhibited cell proliferation and induced apoptosis both in vitro and in vivo. Moreover, the expression of cell cyclerelated genes (including p15, p21, p27 and KLF2) was also analysed after FOXP4-AS1 knockdown. Our findings suggest that IncRNA FOXP4-AS1 plays an important role in colorectal cancer progression and may be a therapeutic target in patients with CRC.

\section{2 | MATERIALS AND METHODS}

\section{1 | Expression profiling data retrieval and analysis of IncRNAs in CRC}

Colorectal cancer IncRNA expression profiling data were retrieved and analysed. Raw microarray data were downloaded from Gene Expression Omnibus (GEO) data sets (GSE21510 and GSE9348). We analysed the IncRNA FOXP4-AS1 expression profiles of colorectal cancer patients included in the GSE21510 and GSE9348 data sets. Paired t-tests based on specific experimental designs were employed to validate probe significance. We used BLAST+ to map these probing sequences to human IncRNA sequences contained in the RefSeq database at NCBI. FDR was calculated by the Benjamini-Hochberg method. The expression levels of FOXP4-AS1 and GAPDH in normal human colorectal tissues were downloaded from BIOGPS data sets (http://biogps.org).

\section{2 | Tissue samples and clinical data collection}

A total of 48 paired CRC and adjacent non-tumour tissue samples were obtained from patients with CRC who underwent surgery at the Second Affiliated Hospital, Nanjing Medical University, between 2013 and 2016. All patients were confirmed to have CRC via histopathological evaluations. None of the patients had been treated before surgery. Detailed information pertaining to clinicopathological characteristics was recorded. All tissue samples were rapidly snap-frozen in liquid nitrogen and stored at $-196^{\circ} \mathrm{C}$ until RNA extraction. Informed consent was obtained from all patients. Our study was approved by the Research Ethics Committee of Nanjing Medical University, China.

\section{3 | RNA extraction and qPCR assays}

Total RNA was extracted from tissues or cultured cells using TRIzol reagent (Invitrogen, Carlsbad, CA, USA), according to the manufacturer's protocol. RNA quantity and quality were determined by a NanoDrop2000 spectrophotometer (Thermo Scientific, Waltham, MA, USA). For qRT-PCR, $1 \mu \mathrm{g}$ of RNA was reverse-transcribed to cDNA using a reverse transcription kit (Takara, Dalian, China). qPCR assays were conducted on an $A B I 7500$. Data were normalized to glyceraldehyde-3-phosphate dehydrogenase (GAPDH) expression. The following primers were used for target amplification: FOXP4-AS1 (forward): 5'-GTGAGCTTCTGGGTTCGACA-3' and FOXP4-AS1 (reverse): 5'-ATTGAGGGTTAGGGCAGCAC -3'; GAPDH (forward): $5^{\prime}$-GG GAGCCAAAAGGGTCAT-3' and GAPDH (reverse): 5'-GAGTCCTT CCACGATACCAA-3'; p15 (forward): 5'-GGACTAGTGGAGAAGGT GCG-3' and p15 (reverse): 5'-GGGCGCTGCCCATCATCATG-3'; p21 (forward): 5'-GTCCACTGGGCCGAAGAG-3' and p21 (reverse): 5'-TGCGTTCACAGGTGTTTCTG-3'; p27 (forward): 5'-TGCA ACCGACGATTCTTCTACTCAA-3' and p27 (reverse): 5' -CAAGCAGTG ATGTATCTGATAAACAAGG-3'; and KLF2 (forward): 5'-CTGCACATG AAACGGCACAT-3' and KLF2 (reverse): $5^{\prime}$-CAGTCACAGTTTGGGAG GGG-3'. All qPCR data were calculated and expressed relative to threshold cycle (shown as $\triangle \mathrm{CT}$ ) values and then converted to fold changes.

\subsection{Cell lines and culture conditions}

Five CRC cell lines (DLD-1, HT-29, HCT116, SW480 and Lovo) were purchased from the Institute of Biochemistry and Cell Biology of the Chinese Academy of Sciences (Shanghai, China). The cells were cultured in RPMI 1640 or DMEM (GIBCO-BRL) medium supplemented with $10 \%$ foetal bovine serum (10\% FBS), $100 \mathrm{U} / \mathrm{mL}$ penicillin and $100 \mathrm{mg} / \mathrm{mL}$ streptomycin in humidified air at $37^{\circ} \mathrm{C}$ with $5 \% \mathrm{CO}_{2}$. 


\section{5 | Cell transfection}

CRC cells were transfected with siRNA oligonucleotides with plasmids using Lipofectamine 2000 (Invitrogen), according to the manufacturer's protocol. The siRNAs were purchased from Invitrogen (Invitrogen). The nucleotide sequences of the siRNAs used for the experiment were as follows: si-FOXP4-AS1 (UGUCGAACCCAGAAGCUCACUUUCC) and si-NC (UUCUCCGAACGUGUCACGUTT). After $48 \mathrm{~h}$ of transfection, the cells were harvested for further study.

\subsection{Cell viability and colony formation assay}

Cell viability was monitored using Cell Proliferation Reagent Kit I (MTT; Roche Applied Science, Roche, Basel, Switzerland). The five CRC cell lines were transfected with si-FOXP4-AS1 or si-NC (3000 cells/well) and cultured in 96-well plates with six replicate wells. Cell viability was assessed according to the manufacturer's recommendations. For the colony formation assay, a total of 500 cells were seeded in a six-well plate and maintained in medium containing 10\% FBS, which was replaced every 5 days. After 2 weeks, the cells were fixed with methanol and stained with $0.1 \%$ crystal violet (Sigma-Aldrich, St. Louis, MO, USA). Visible colonies were counted manually. Wells were measured in triplicate in each treatment group.

\section{7 | Flow cytometry}

DLD-1, HT-29 and HCT116 cells transfected with si-FOXP4-AS1 or si-NC were harvested after $48 \mathrm{~h}$, stained with PI using a Cycletest ${ }^{\mathrm{TM}}$ Plus DNA Reagent Kit (BD Biosciences, Franklin Lakes, NJ, USA), according to the manufacturer's protocol, and analysed with a flow cytometer (FACScan ${ }^{\circledR}$; BD Biosciences) equipped with CellQuest software (BD Biosciences). The percentages of cells in G0/G1, S and $\mathrm{G} 2 / \mathrm{M}$ phase were calculated and compared.

DLD-1, HT-29 and HCT116 cells transfected with si-FOXP4-AS1 or si-NC were harvested after $48 \mathrm{~h}$ for apoptosis analysis. The cells were then treated with fluorescein isothiocyanate (FITC)-Annexin $\mathrm{V}$ and propidium iodide (PI) in the dark at room temperature, according to the manufacturer's recommendations. The cells were subsequently analysed by FACScan ${ }^{\circledR}$ and identified as viable, dead, early apoptotic or late apoptotic cells.

\section{8 | Ethynyl deoxyuridine (Edu) analysis}

Proliferating cells were assessed using a 5-ethynyl-2-deoxyuridine labelling/detection kit (Ribobio, Guangzhou, China), according to the manufacturer's protocol. Briefly, DLD-1, HT-29 and HCT116 cells were cultured in 96-well plates at a density of $5 \times 10^{3}$ cells per well and transfected with si-FOXP4-AS1 or si-NC for $48 \mathrm{~h}$. Then, $50 \mu \mathrm{M}$ Edu labelling medium was added to the cell culture, which was incubated for $2 \mathrm{~h}$ at $37^{\circ} \mathrm{C}$ with $5 \% \mathrm{CO}_{2}$. Then, the cultured cells were fixed with $4 \%$ paraformaldehyde $(\mathrm{pH} \mathrm{7.4)}$ for 30 min and treated with $0.5 \%$ Triton X-100 for 20 min at room temperature. After being washed with PBS, the samples were stained with anti-Edu working solution for $30 \mathrm{~min}$ at $25^{\circ} \mathrm{C}$ before being incubated with $100 \mu \mathrm{L}$ of DAPI $(5 \mu \mathrm{g} /$ $\mathrm{mL}$ ) for $30 \mathrm{~min}$ at $25^{\circ} \mathrm{C}$ and observed under a fluorescent microscope. The percentage of Edu-positive cells was calculated from five random fields in three wells.

\subsection{Tumour xenografts in animals}

Four-week-old athymic male mice were purchased from the Animal Center of Nanjing University (Nanjing, China) and maintained in pathogen-free conditions. DLD-1 cells were transfected with shFOXP4-AS1 or empty vector and harvested from six-well plates, washed with phosphate-buffered saline (PBS), and resuspended at a density $2 \times 10^{7}$ cells $/ \mathrm{mL}$. Each mouse was subsequently injected in the lower right flank with $100 \mu \mathrm{L}$ of suspended cells. Tumour growth was examined every 3 days, and tumour volumes were measured as the product of length $\times$ width $^{2} \times 0.5$. At 15 days post-injection, the mice were sacrificed via $\mathrm{CO}_{2}$ asphyxiation, and tumour growth was examined.

\subsection{0 | Immunohistochemistry (IHC)}

Xenograft tumours were immunostained for $\mathrm{H} \& \mathrm{E}$ and Ki-67. Signals were amplified and visualized with $3^{\prime}$-diaminobenzidine chromogen, and the tumours were subsequently counterstained with haematoxylin. Expression was considered positive when $60 \%$ or more of the tumour cells were stained. The IHC staining results were independently scored by the author and a pathologist to minimize subjectivity and then compared, and final comprehensive results were obtained. AntiKi-67 (1:100) antibodies were purchased from R \& D.

\subsection{1 | Statistical analysis}

All statistical analyses were performed using SPSS software, version 22.0 (SPSS, Chicago, IL, USA). Student's $t$ test or a chi-square test was used to evaluate significant differences between groups of data. All data are represented as the mean \pm SD. $P<.05$ was considered significant. ${ }^{*} P<.05 ;{ }^{* *} P<.01$

\section{3 | RESULTS}

\section{1 | IncRNA FOXP4-AS1 expression levels in CRC}

In an attempt to identify novel oncogenic IncRNAs that are involved in CRC progression, two microarray data sets (GSE21510 and GSE9348) were used to analyse IncRNAs that were differentially expressed in colorectal tumours samples and corresponding nontumour samples. We found that IncRNA FOXP4-AS1 was consistently up-regulated in both the GSE21510 and the GSE9348 data sets (Figure 1E). The FOXP4-AS1 gene is located at the chromosomal locus 6p21.1 and encodes a 588 bp transcript (Figure 1A). In addition, BioGPS data set analysis indicated that the FOXP4-AS1 gene is not expressed in normal human colorectal tissues (A zscore $>5$ suggests that a gene is expressed in a particular tissue) 
(A) 1 ccctggtttt ctgtggaaag tgagcttctg ggttcgacag tgggaccggc acagacttc 61 ccgcagctac aggccatacg acaaccccgc tgctctttct ttctgcgggc actcgggcca 121 gtcctaacaa ttgcccctca agctgtgtgt gctgccctaa ccctcaatcc cgaacccgaa 181 acagtgcgtg ctgggacgaa tctaggcagg tctccgaaac cggggtgaag atgctgaagt 241 tcaggtcatt ccggactcct gacgccgcca gtcccgcggt gagacgtgag cgecattggc 301 gtccgtggce tctgtttccg tggcaaccta gtaaccatta atttcaatt aaaggagaca 361 aaaagctcga tgacagctcc aggtctgctg aagatgtcaa gaatctgtat taatatacag 421 caagagagca taattgtgtg tccatcttcc agagcagcga aaaatggagg gataattacc 481 agctcgaaag ccactctgta atttagttct tactgtcacc acagccctac acattagcat 541 aaattaaaag cagagtttat tgttatcaaa gtgcaaaaaa aaaaaaaa

(C)

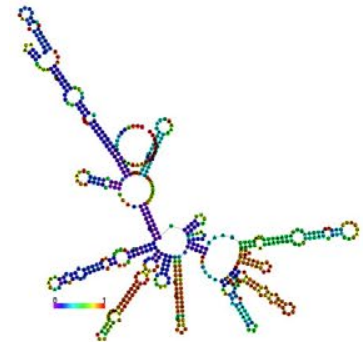

MFE secondary structure

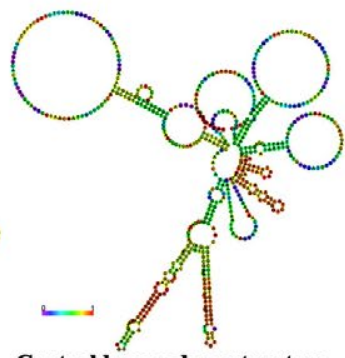

Centrold secondary structure
(B) FOXP4-AS1

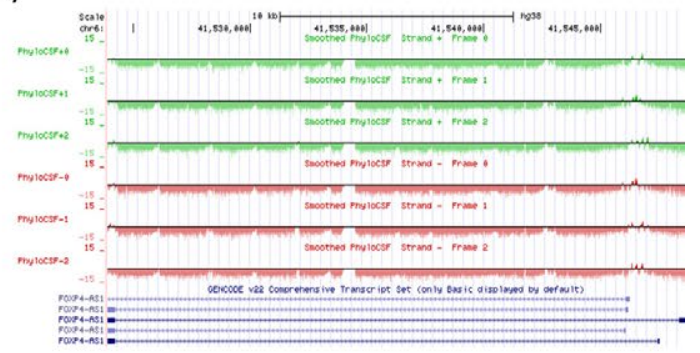

GAPDH

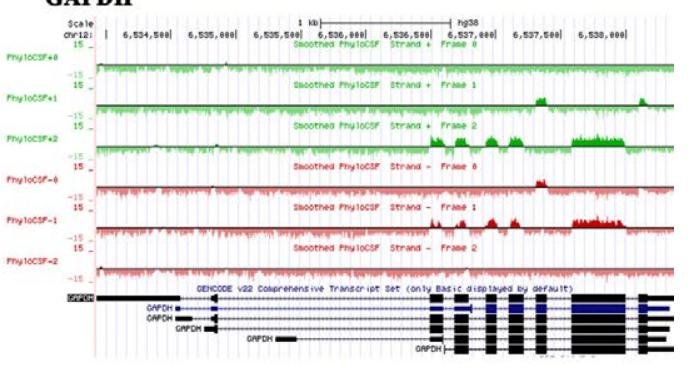

(D)

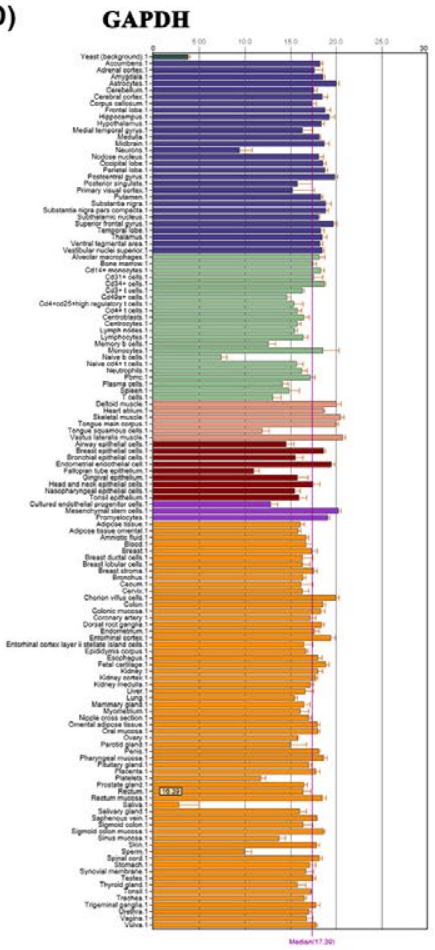

(E)

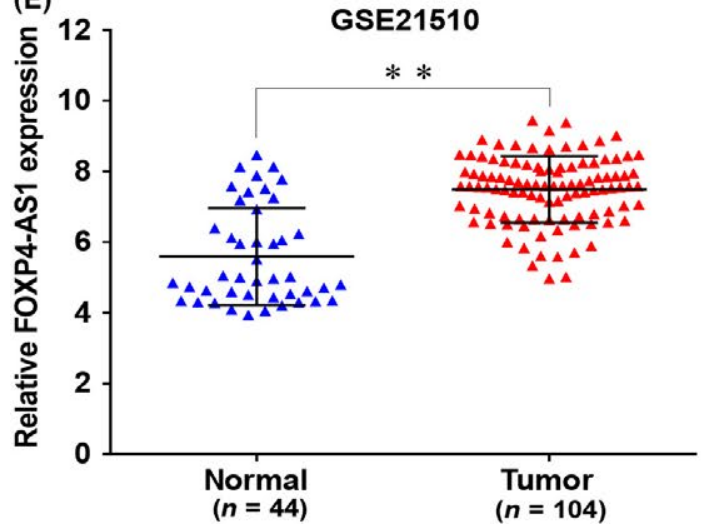

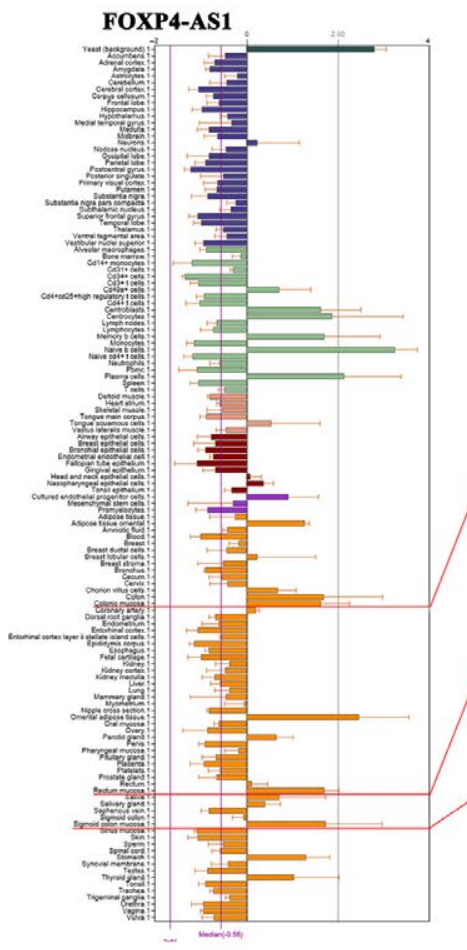

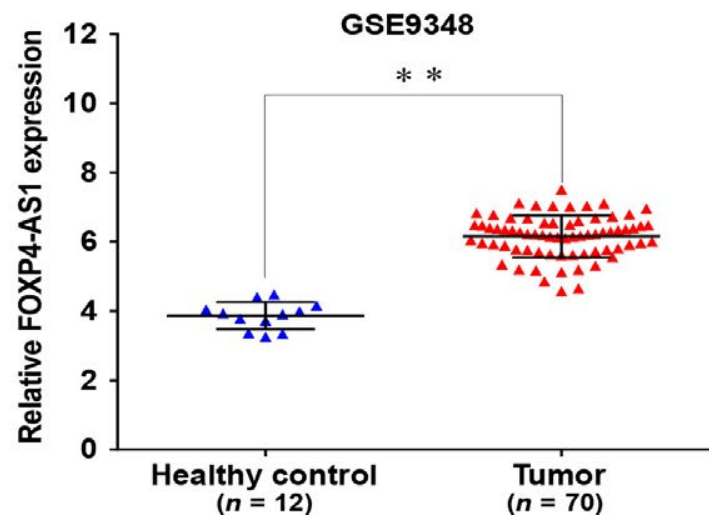

z-score

Colon 1.69

Colonic mucosa 1.63

Rectum 0.91

Rectum mucosa 1.70

Sigmoid colon $\mathbf{- 0 . 0 5}$

Sigmoid colon mucosa 1.73 $(n=12)$

$(n=70)$ 
FIGURE 1 Higher FOXP4-AS1 expression levels in CRC and coding potential analyses of FOXP4-AS1 transcripts. A, The full sequence of FOXP4-AS1 is published in the NCBI database (NR_126415.1). B, PhyloCSF predicted that FOXP4-AS1 has no protein coding potential. The tracks showed the PhyloCSF scores for each codon in each of six frames. Regions with a score greater than 0 were predicted to be coding regions, while regions with a score less than 0 were predicted to be non-coding regions. The protein-coding gene GAPDH was used as a control. C, Prediction of the structure of FOXP4-AS1 based on minimum free energy (MFE) and partition function. The colour scale indicates the confidence for the prediction for each base, with shades of red indicating strong confidence (http://rna.tbi.univie.ac.at/). D, Low FOXP4-AS1 expression levels in normal human colorectal tissues. The protein-coding gene GAPDH was used as a control. E, Higher FOXP4-AS1 expression levels in the profiles of CRC patient tissue samples from Gene Expression Omnibus

(A)

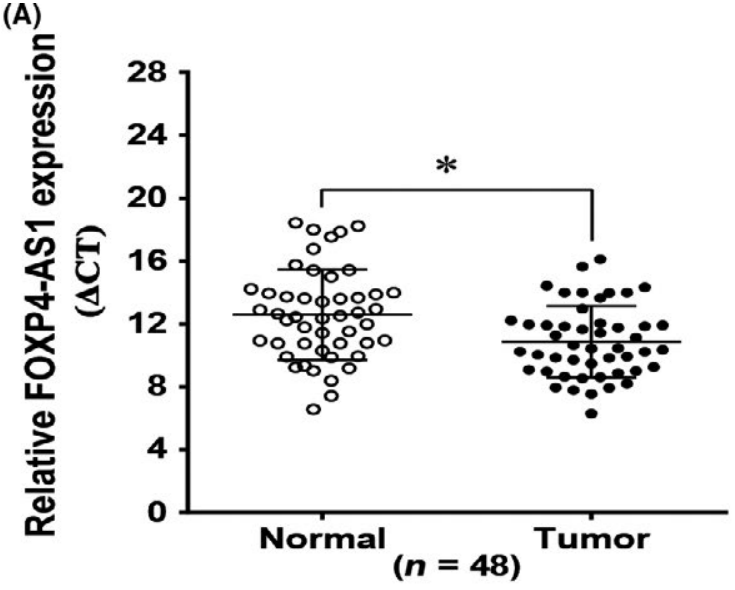

(B)

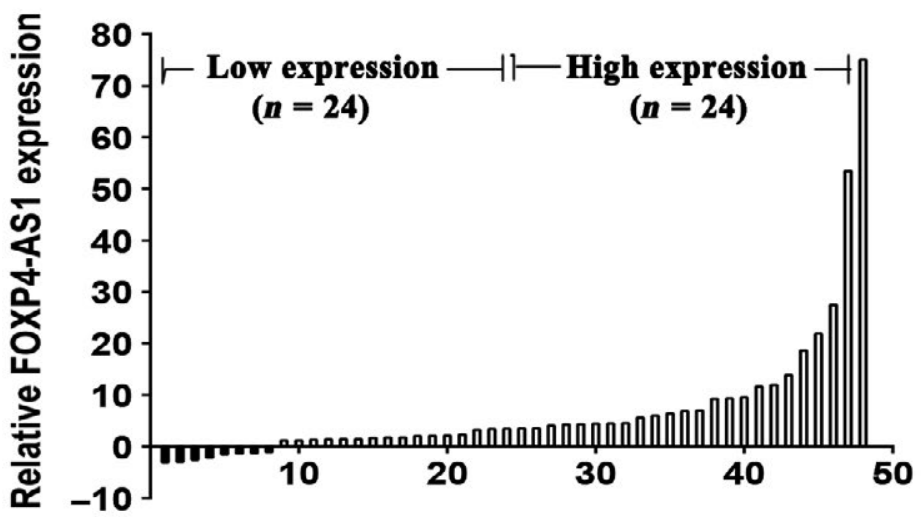

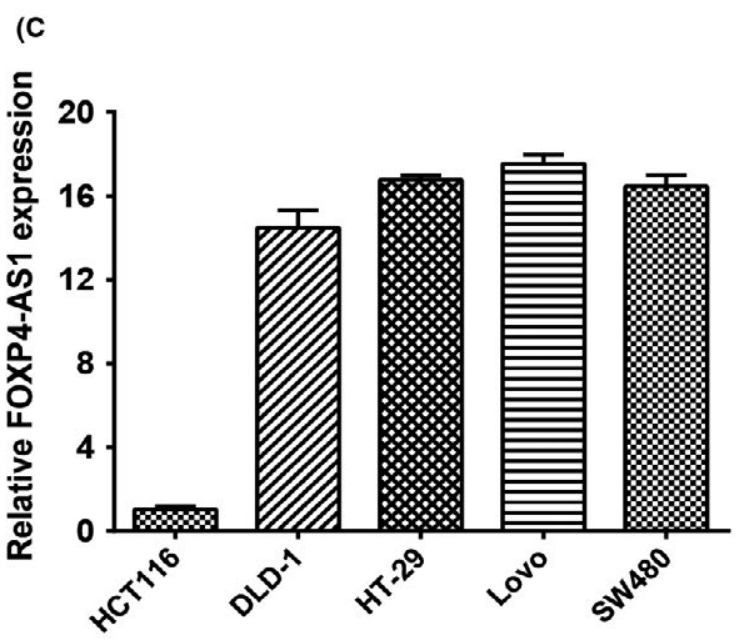

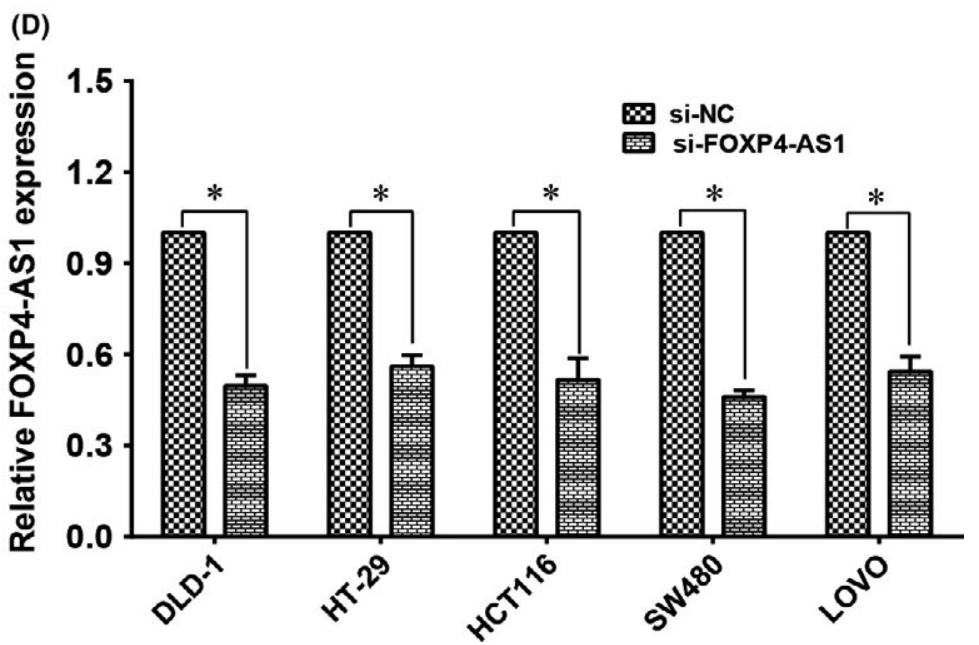

FIGURE 2 Relative FOXP4-AS1 expression levels in CRC tissues and CRC cell lines. A, FOXP4-AS1 expression levels in matched tumour tissues and adjacent normal tissues from 48 CRC patients were measured by qPCR. Relative gene expression determinations were made with the comparative delta CT normalized to GAPDH expression $(P<.05)$. B, Patients were divided into two groups according to the median value of relative FOXP4-AS1 expression. C, qPCR analysis of FOXP4-AS1 expression levels in five CRC cell lines (HCT116, DLD-1, HT-29, Lovo and SW480). D, qPCR analysis of FOXP4-AS1 expression levels in five CRC cell lines transfected with siRNA. Bars: s.d., ${ }^{*} P<.05,{ }^{* *} P<.01$, from three independent experiments

(Figure 1C). Moreover, PhyloCSF predicted that FOXP4-AS1 has no protein-coding potential. The tracks showed the PhyloCSF score for each codon in each of six frames. Regions with a score greater than 0 were predicted to be coding regions, while regions with a score less than 0 were predicted to be non-coding regions. The proteincoding gene GAPDH was used as a control (Figure 1B). These results indicate that IncRNA FOXP4-AS1 expression is increased in CRC tissues and that FOXP4-AS1 may serve as an oncogene in CRC progression.

\section{2 | FOXP4-AS1 overexpression was positively correlated with advanced pathological stages and larger tumour sizes in patients with CRC}

To confirm that FOXP4-AS1 was overexpressed in CRC tissues, we detected FOXP4-AS1 expression levels in 48 paired CRC samples and analysed adjacent normal tissues with qPCR. All samples were normalized to GAPDH. The results showed that FOXP4-AS1 expression levels were significantly higher in tumour tissues than in adjacent 
TABLE 1 Correlation between FOXP4-AS1 expression and clinicopathologic characteristics of patients with CRC ( $n=48)$.

\begin{tabular}{|c|c|c|c|}
\hline \multirow[b]{2}{*}{ Characteristics } & \multicolumn{2}{|l|}{ FOXP4-AS1 } & \multirow{2}{*}{$\begin{array}{l}P \\
\text { Chi-squarec } \\
\text { test, } P \text { value }\end{array}$} \\
\hline & $\begin{array}{l}\text { Low No. of } \\
\text { cases }\end{array}$ & $\begin{array}{l}\text { High No. of } \\
\text { cases }\end{array}$ & \\
\hline \multicolumn{4}{|l|}{ Age (years) } \\
\hline$\leq 60$ & 8 & 4 & \multirow[t]{2}{*}{0.318} \\
\hline$>60$ & 16 & 20 & \\
\hline \multicolumn{4}{|l|}{ Gender } \\
\hline Male & 15 & 17 & \multirow[t]{2}{*}{0.760} \\
\hline Female & 9 & 7 & \\
\hline \multicolumn{4}{|l|}{ Tumor size (cm) } \\
\hline$\leq 5$ & 13 & 8 & \multirow[t]{2}{*}{0.036} \\
\hline$>5$ & 11 & 19 & \\
\hline \multicolumn{4}{|c|}{ Histologic differentiation } \\
\hline Well & 9 & 5 & \multirow[t]{2}{*}{0.341} \\
\hline Poorly & 15 & 19 & \\
\hline \multicolumn{4}{|l|}{ TNM stage } \\
\hline $\mathrm{I} / \mathrm{II}$ & 16 & 8 & \multirow[t]{2}{*}{0.042} \\
\hline III/IV & 8 & 16 & \\
\hline \multicolumn{4}{|c|}{ Lymph node metastasis } \\
\hline Positive & 13 & 9 & \multirow[t]{2}{*}{0.385} \\
\hline Negative & 11 & 15 & \\
\hline \multicolumn{4}{|c|}{ Primary tumor site } \\
\hline Colon & 10 & 16 & \multirow[t]{2}{*}{0.147} \\
\hline Rectum & 14 & 8 & \\
\hline
\end{tabular}

normal tissues $(P<.01$; Figure $2 A)$. Then, to investigate the relationship between FOXP4-AS1 expression and clinicopathological features, we divided the samples into high (above the median, $\mathrm{n}=24$ ) and low (below the median, $\mathrm{n}=24$ ) FOXP4-AS1 expression groups according to the median FOXP4-AS1 level (Figure 1B). A chi-square test was performed to compare the clinicopathological features of the two groups. As shown in Table 1, FOXP4-AS1 overexpression in CRC tissues was significantly correlated with larger tumour sizes $(P=.036)$ and advanced TNM stages $(P=.042)$. However, several other clinical parameters were found to be non-significantly associated with FOXP4-AS1 expression (Table 1).

\section{3 | Modulation of FOXP4-AS1 expression in CRC cells}

We next performed qPCR analysis to examine FOXP4-AS1 expression in a panel of CRC cell lines, including DLD-1, HT-29, HCT116, SW480 and Lovo. We found that FOXP4-AS1 expression levels were higher in DLD-1 $(P<.01)$, HT-29 $(P<.01)$, SW480 $(P<.01)$ and Lovo $(P<.01)$ cells than in HCT116 cells (Figure $2 \mathrm{C}$ ). Then, to investigate the functional effects of FOXP4-AS1 dysregulation in CRC cells, we knocked down its expression via FOXP4-AS1 siRNA transfection. qPCR analysis of FOXP4-AS1 levels was performed $48 \mathrm{~h}$ post-transfection. The results showed that FOXP4-AS1 expression was significantly reduced by siFOXP4-AS1 transfection compared with control cells (Figure 2D).

\subsection{FOXP4-AS1 promoted CRC cell proliferation}

To assess the biological role of FOXP4-AS1 in CRC cells, we performed an MTT assay. The results showed that FOXP4-AS1 knockdown significantly inhibited cell viability all in five CRC cell lines compared with control cells (Figure 3A). Similarly, the colony formation assay results showed that clonogenic survival was strikingly decreased following FOXP4-AS1 inhibition all in five CRC cell lines (Figure 3B).

Next, to determine whether the effects of FOXP4-AS1 on CRC cell proliferation are the result of FOXP4-AS1-mediated changes in cell cycle progression, we performed flow cytometry assay using DLD-1, HT-29 and HCT116 cells. The results of si-FOXP4-AS1 or si-NC transfection for $48 \mathrm{~h}$ showed that FOXP4-AS1 knockdown increased the percentage of cells in G0/G1 phase and decreased the percentage of cells in $\mathrm{S}$ and G2/M phase compared to control cells (Figure 4A). EdU analysis yielded similar results (Figure 5A). Previous studies have demonstrated that cell cycle-related genes can be regulated by IncRNAs, ${ }^{19,20}$ and our previous study showed that $\mathrm{p} 21$ serves as a tumour suppressor in CRC. ${ }^{18,21}$ To determine whether the inhibition of cell cycle-related genes (including p15, p21, p27 and KLF2) is required for the above-mentioned FOXP4AS1-mediated effects on CRC cell proliferation, we performed qPCR analysis. The results showed that FOXP4-AS1 inhibition leads to increases in p15, p21, p27 and KLF2 expression in four CRC cell lines (Figure 5B). These data indicate that FOXP4-AS1 promotes CRC cell proliferation by promoting cell cycle progression.

\section{5 | FOXP4-AS1 knockdown induced CRC cell apoptosis}

To determine whether the effects of FOXP4-AS1 on colorectal cancer cell proliferation are associated with cell apoptosis inhibition, we performed flow cytometry assays. The results showed that DLD-1, HT29 and HCT116 cells transfected with FOXP4-AS1 siRNA had higher apoptosis rates than control cells (Figure 4B). These data demonstrate that FOXP4-AS1 promotes the proliferation phenotype and inhibits the apoptosis of colorectal cancer cells.

\section{6 | FOXP4-AS1 knockdown inhibits CRC cell tumourigenesis in vivo}

To confirm the impact of FOXP4-AS1 on CRC cell growth in vivo, DLD-1 cells transfected with sh-FOXP4-AS1 or empty vector were injected into male nude mice. The cells were transfected with empty vector as a control. At 15 days post-injection, tumour growth in the sh-FOXP4-AS1 group was markedly slower than that in the control group (Figure 6A). Correspondingly, tumour volumes and weights were obviously decreased in transfected cells compared with control cells (Figure 6B,C). Moreover, immunohistochemistry (IHC) analysis confirmed that tumours that formed from DLD-1/sh-FOXP4-AS1 cells exhibited lower Ki-67 staining than those that formed from 

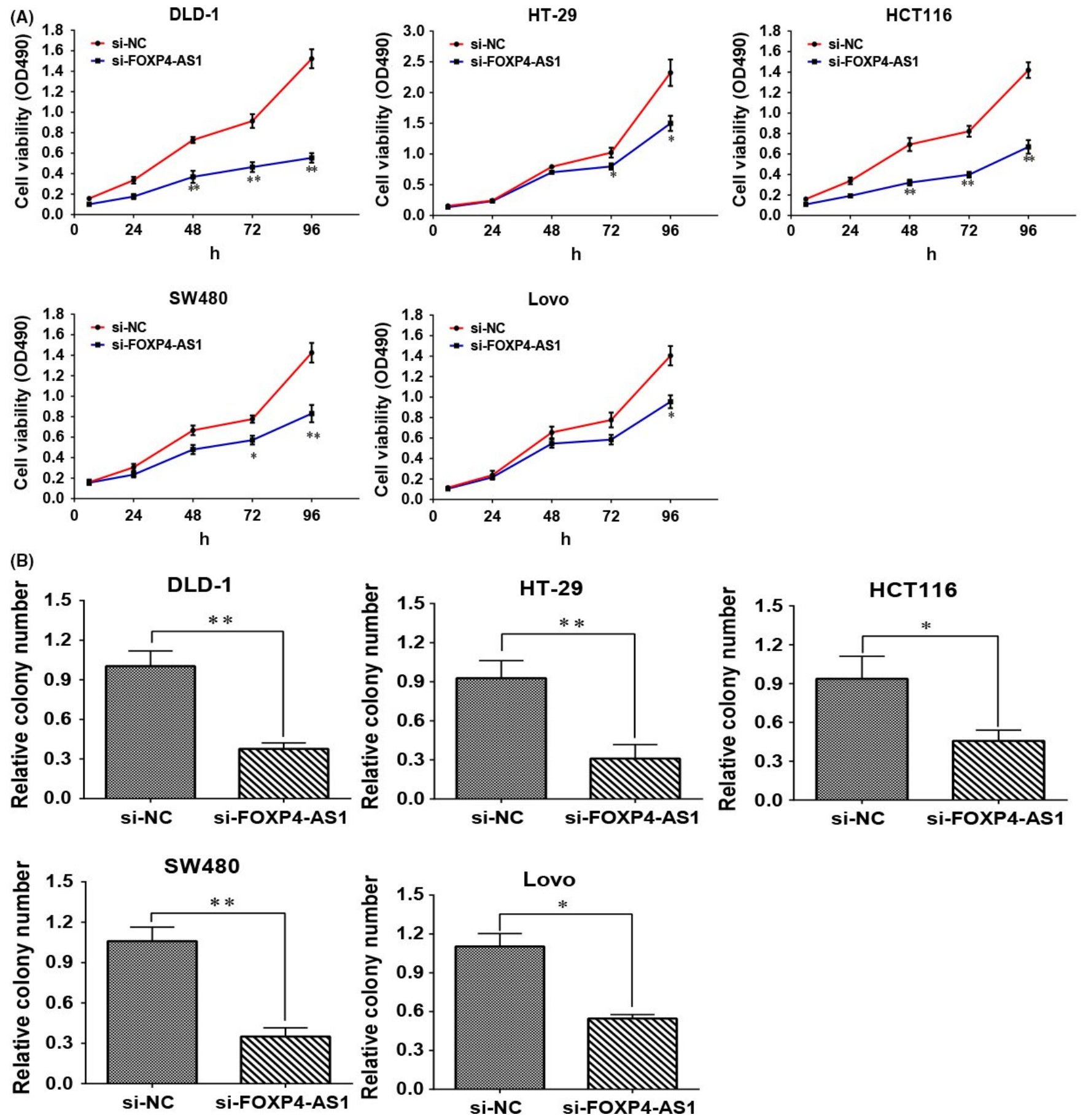

FIGURE 3 Effect of FOXP4-AS1 on CRC cell viability in vitro. A, MTT assays were used to determine the cell viability of five CRC cell lines transfected with siRNAs against FOXP4-AS1. B, Colony-forming assays were conducted to assess the proliferation of five CRC cell lines transfected with siRNAs against FOXP4-AS1. Bars: s.d., ${ }^{*} P<.05,{ }^{* *} P<.01$, from three independent experiments

control cells (Figure 6E). Our results indicate that FOXP4-AS1 knockdown suppresses colorectal cancer cell tumour growth in vivo.

\section{4 | DISCUSSION}

Cancer is a disease of the genome caused by oncogene activation and tumour suppressor gene inhibition. In recent years, deep sequencing studies including large consortia (such as ENCODE project, TCGA and ICGC) and advances in technology (such as tiling arrays and RNA deep sequencing) have enabled the identification of numerous tumourspecific mutations not only in protein-coding sequences but also in non-coding sequences. ${ }^{4,22}$ In particular, long non-coding RNAs (IncRNAs) have received increased attention. LncRNAs are strikingly similar to mRNAs. They are RNA polymerase II transcripts that are capped, spliced and polyadenylated and are devoid of an open reading frame 
(A)

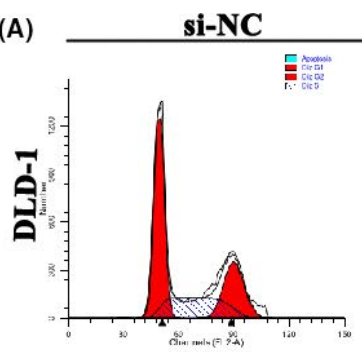

(A)
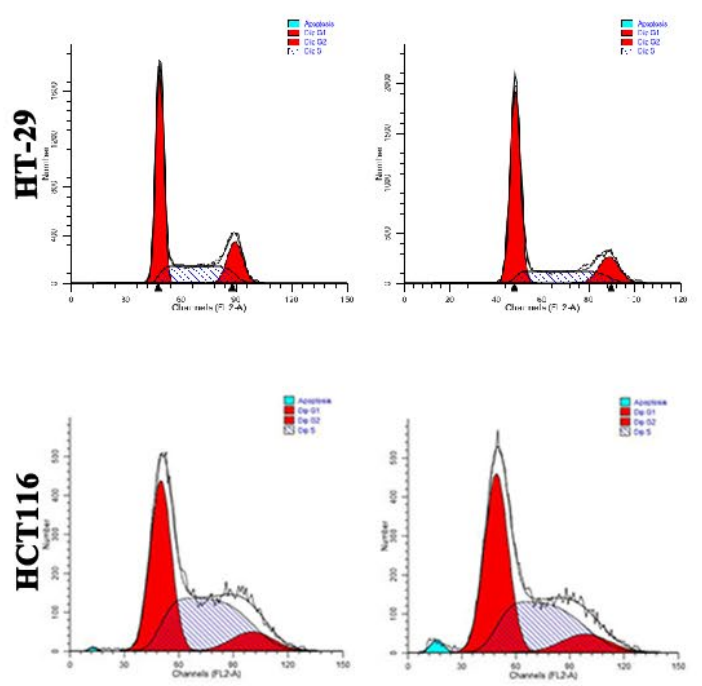

(B)

si-NC
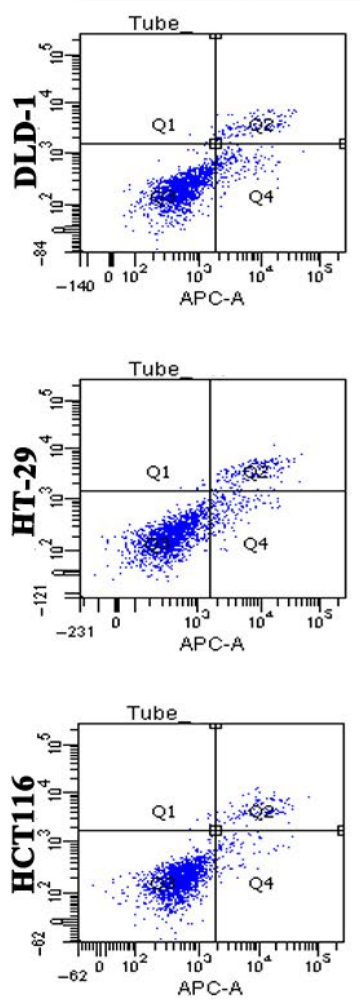

si-FOXP4-AS1

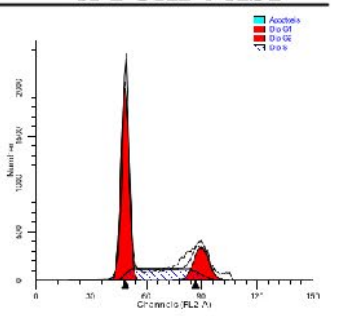

si-FOXP4-AS1

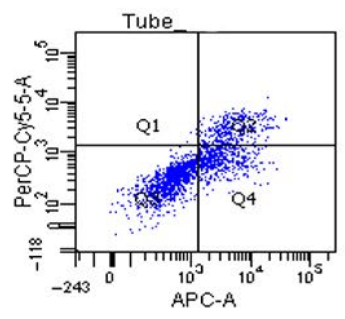

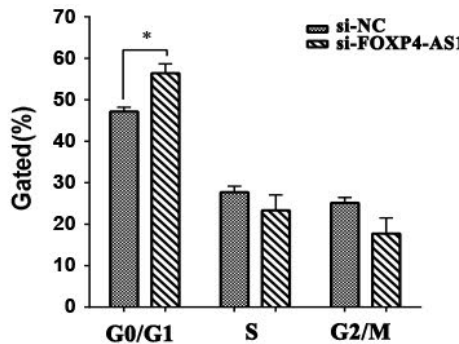
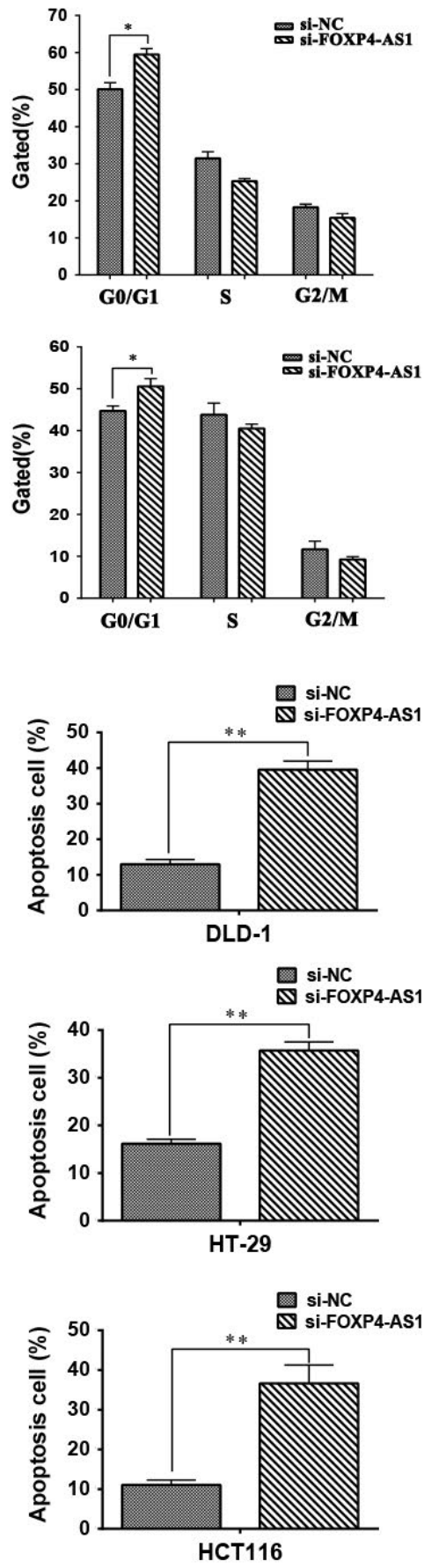

FIGURE 4 Effect of FOXP4-AS1 on CRC cell cycle progression and apoptosis in vitro. A, DLD-1, HT-29 and HCT116 cell cycle progression was analysed by flow cytometry. The bar chart represents the percentages of cells in G1-G0, S or G2-M phase, as indicated. B, DLD-1, HT29 and HCT116 cells were stained and analysed by flow cytometry. Q2, early apoptotic cells. Q4, terminal apoptotic cells. Bars: s.d., ${ }^{*} P<.05$, 
(A)
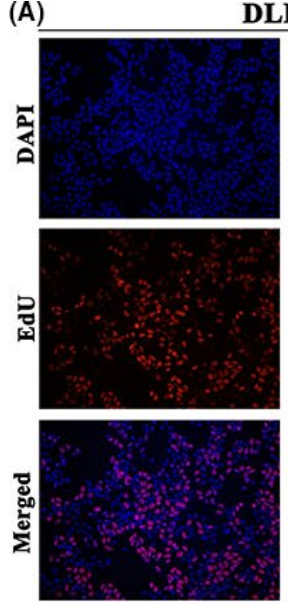

si-NC
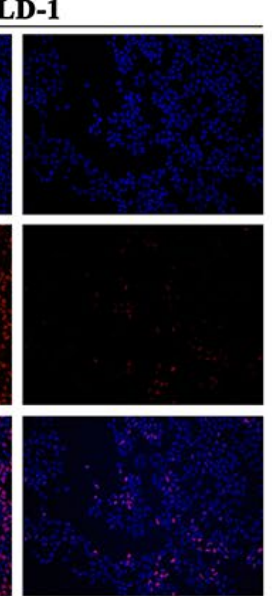

si-FOXP4-AS1

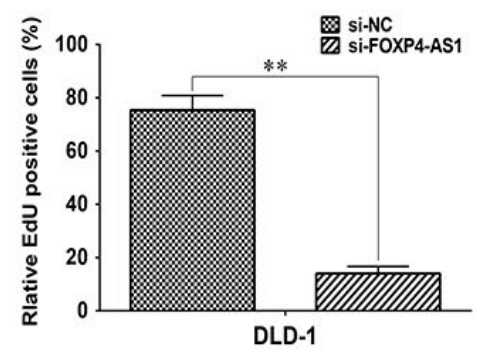

(B)
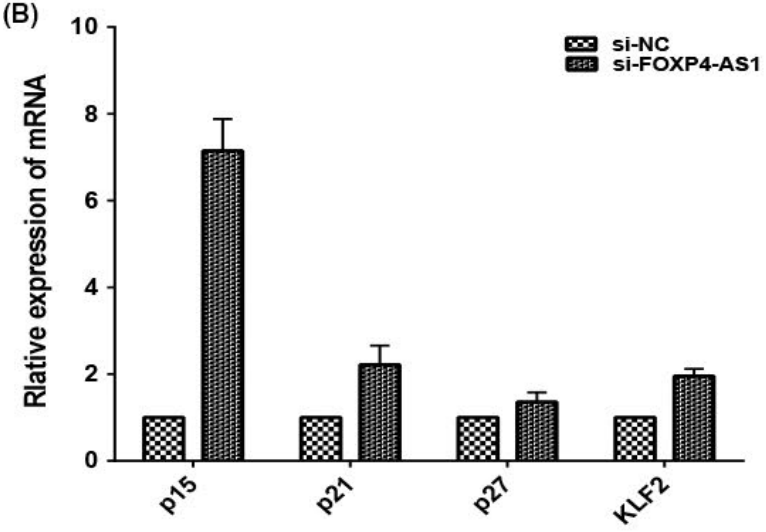

DLD-1

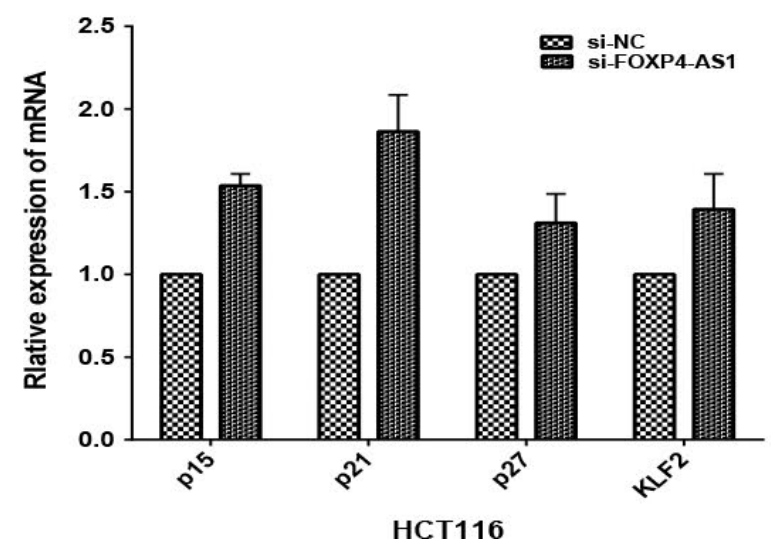

HT-29
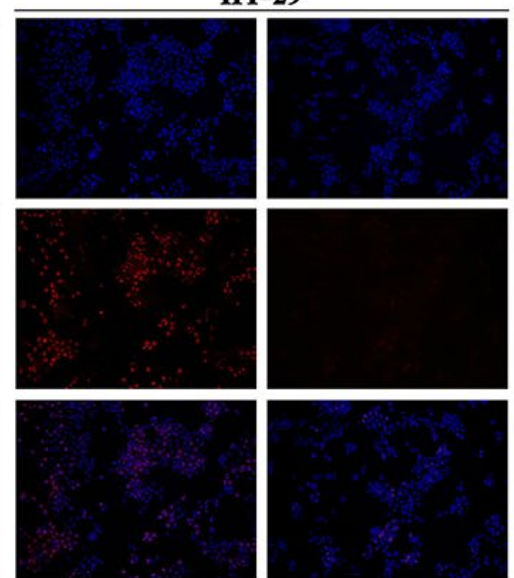

si-NC

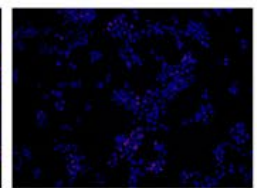

si-FOXP4-AS1

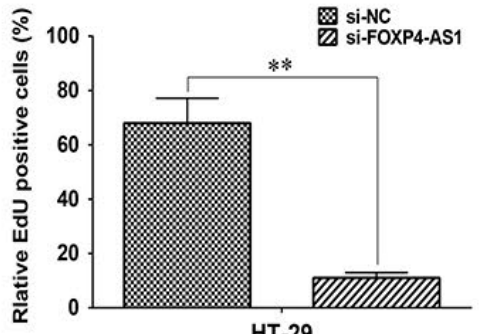

HT-29
HCT116
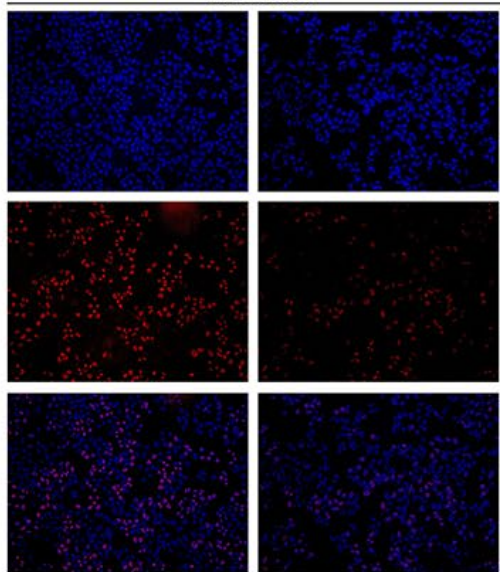

si-NC

si-FOXP4-AS1

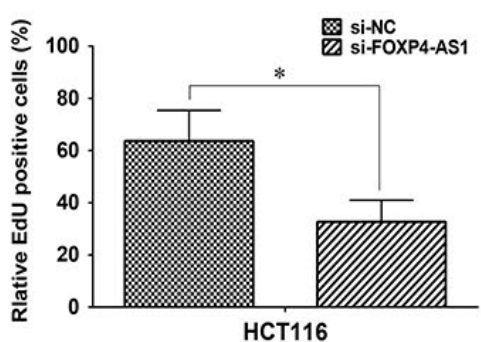

HCT116

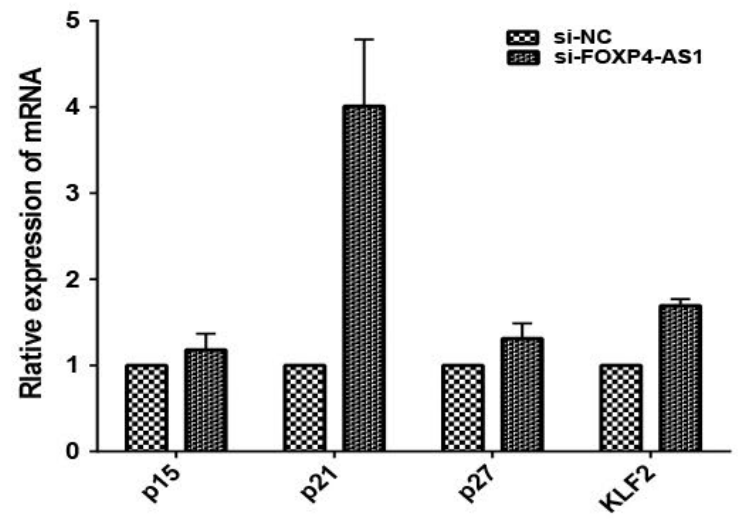

HT-29

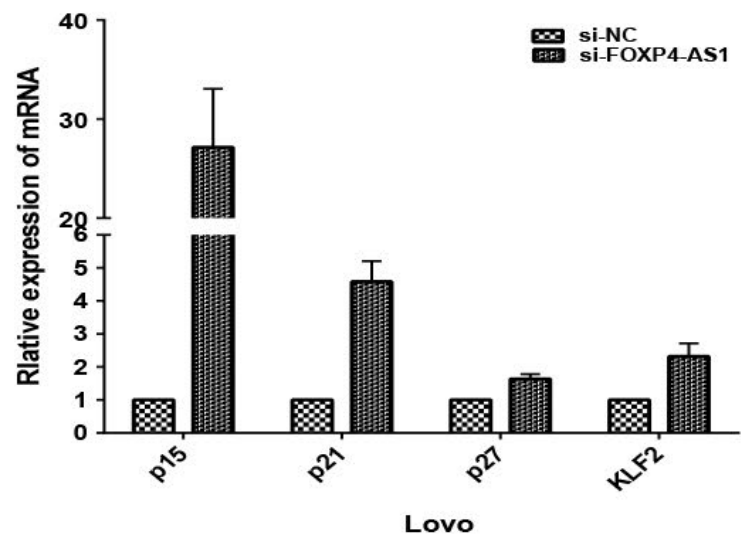

FIGURE 5 FOXP4-AS1 is required to suppress p15, p21, p27 and KLF2 expression and thus regulate the CRC cell cycle and CRC proliferation. A, Effect of FOXP4-AS1 on CRC cell proliferation, as shown by EdU assay. Proliferating CRC cells were labelled with Edu. The Click-it reaction revealed Edu staining (red). Cell nuclei were stained with DAPI (blue). Representative images and data based on three independent experiments. B, p15, p21, p27 and KLF2 mRNA expression levels were determined by qPCR following FOXP4-AS1 knockdown Bars: $S D,{ }^{*} P<.05,{ }^{* *} P<.01$ 
(A)

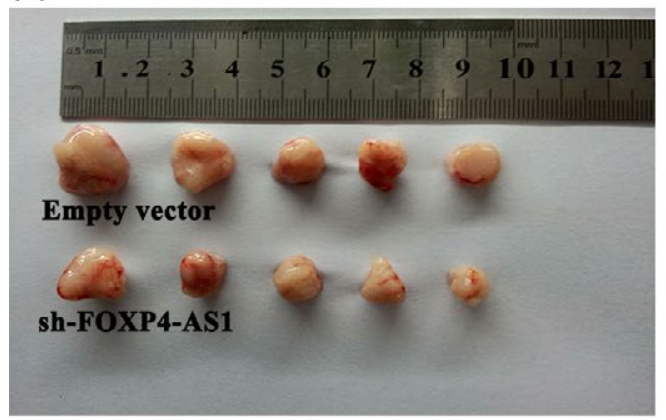

(D)

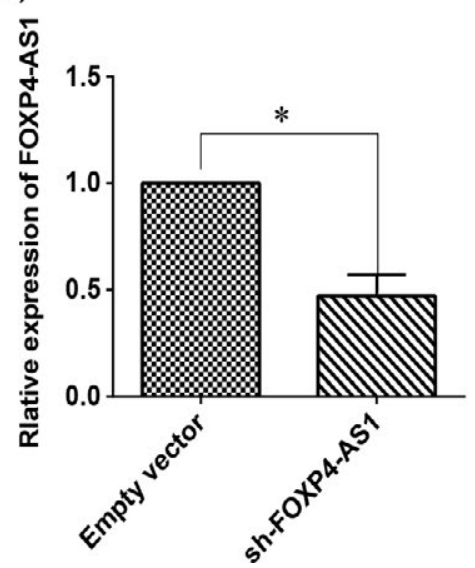

(B)

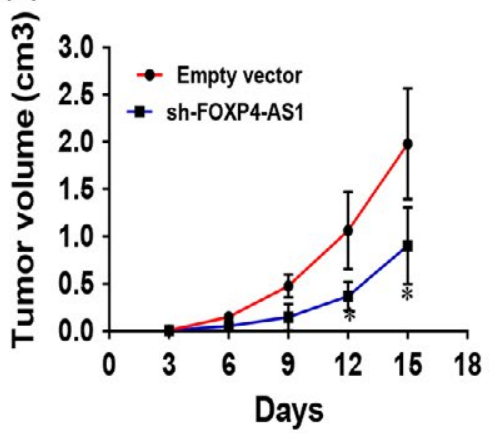

(E)

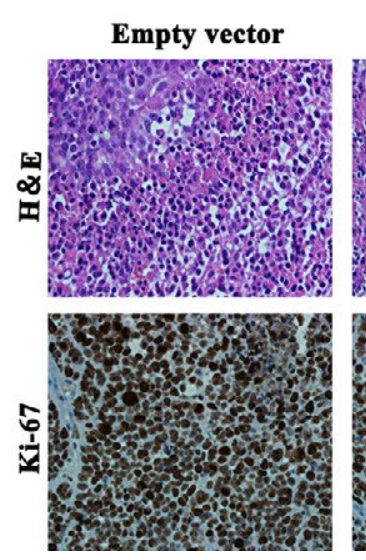

(C)

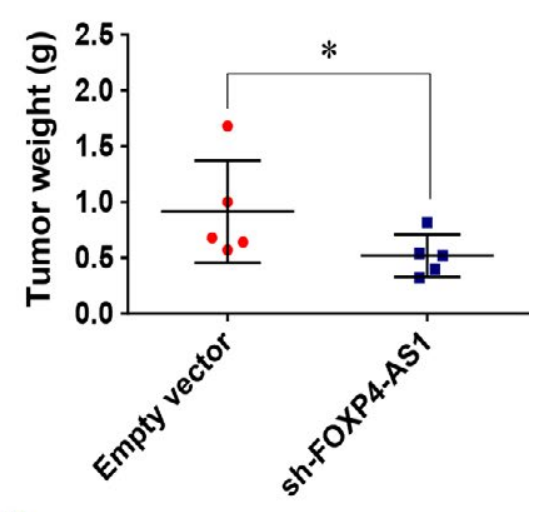

FIGURE 6 FOXP4-AS1 knockdown inhibits tumour growth in a xenograft mouse model (A) Tumours were harvested 15 days after the injection of DLD-1 cells transfected with sh-FOXP4-AS1 or empty vector. (B, C) Tumour volumes were calculated every 3 days after injection. Tumour weights were measured when the xenograft tumours were harvested. D, qPCR analysis of FOXP4-AS1 expression in xenograft tumour tissues. E, HE staining and immunohistochemistry images of the xenograft tumours. Representative Ki-67 protein levels in the xenograft tumours, as determined via IHC. Bars: SD, ${ }^{*} P<.05,{ }^{* *} P<.01$

(ORF) that can be translated into a protein sequence. ${ }^{23}$ More recently, studies have reported that some IncRNAs are aberrantly expressed in and associated with the onset and progression of a variety of cancers, especially CRC. ${ }^{24,25}$ These IncRNAs have gained great clinical value as new biomarkers of and novel therapeutic targets for this deadly disease. $^{26,27}$ For example, Huang et al. demonstrated that IncRNA CASC2 plays an important role in the pathobiology of human CRC and that it can function as a ceRNA by competitively binding miR-18a, thereby relieving the suppression of PIAS3 by miR-18a in CRC cells. ${ }^{28}$ Wan et al. found that IncRNA HOTAIRM1 and CEA down-regulation is associated with CRC and that the combined assay of these two biomarkers appears to be promising with respect to the diagnosis of CRC (sensitivity was increased to $84 \%$ ). ${ }^{29}$ Our previous study demonstrated that HOTTIP promotes colorectal cancer cell proliferation partly via p21 silencing. ${ }^{21}$

In the present study, we investigated the potential role of FOXP4-AS1 as an oncogene in CRC development. First, two colorectal cancer gene expression data sets were obtained from the indicated publicly available GEO databases (GSE21510 and GSE9348). We found IncRNA FOXP4-AS1 was consistently up-regulated in both the GSE21510 and the GSE9348 data sets. Our subsequent studies of clinical samples and CRC cell lines demonstrated that FOXP4-AS1 is up-regulated and that FOXP4-AS1 overexpression is significantly associated with advanced pathological stages and larger tumour sizes in patients with CRC. As sustained proliferative signalling, growth suppressor evasion, and cell death resistance are the basic hallmark capabilities of malignancies, we evaluated the effects of FOXP4-AS1 on CRC cell proliferation and apoptosis. ${ }^{30,31}$ The results showed that FOXP4-AS1 knockdown inhibited cell proliferation and tumour growth both in vitro and in vivo by extending the G0/G1-S phase transition. These findings suggest that FOXP4-AS1 plays an important role in modulating CRC progression. Moreover, flow cytometry assays demonstrated that the effects of FOXP4-AS1 on colorectal cancer cell proliferation were associated with cell apoptosis inhibition. We also analysed cell cycle-related gene expression after FOXP4-AS1 knockdown. Further investigation is required to understand the mechanism underlying the relationship between FOXP4-AS1 and cell cyclerelated gene expression in colorectal cancer.

In summary, our study showed for the first time that IncRNA FOXP4-AS1 expression is up-regulated in CRC tissues and that FOXP4-AS1 overexpression is a poor prognostic factor in CRC patients. Furthermore, FOXP4-AS1 knockdown inhibited CRC cell proliferation and induced apoptosis both in vitro and in vivo. Therefore, FOXP4-AS1 functions as an oncogene in CRC tumourigenesis and thus may be an effective therapeutic target in and novel biomarker for CRC. 


\section{ACKNOWLEDGEMENTS}

This work was supported by the Medical Science and Technology Development Foundation, Jiangsu Province Department of Health (H201512), the Six Talents Peak Project of Jiangsu Province (WSN050), the Medical Science and Technology Development Foundation of Nanjing (YKK13178) and the Natural Science Foundation of Jiangsu Province of China (BK20151578).

\section{REFERENCES}

1. Siegel R, Desantis C, Jemal A. Colorectal cancer statistics, 2014. CA Cancer J Clin. 2014;64:104-117.

2. Siegel RL, Miller KD, Jemal A. Cancer statistics, 2016. CA Cancer J Clin. 2016;66:7-30.

3. Harrow J, Frankish A, Gonzalez JM, et al. GENCODE: the reference human genome annotation for The ENCODE Project. Genome Res. 2012;22:1760-1774.

4. Djebali S, Davis CA, Merkel A, et al. Landscape of transcription in human cells. Nature. 2012;489:101-108.

5. Gardini A, Shiekhattar R. The many faces of long noncoding RNAs. FEBS J. 2015;282:1647-1657.

6. Lopez-Pajares V. Long non-coding RNA regulation of gene expression during differentiation. Pflugers Arch. 2016;468:971-981.

7. Blythe AJ, FoxAH, Bond CS. The ins and outs of IncRNA structure: How, why and what comes next? Biochim Biophys Acta. 2016;1859:46-58.

8. Dhamija S, Diederichs S. From junk to master regulators of invasion: IncRNA functions in migration. EMT and metastasis. Int J Cancer. 2016;139:269-280

9. Xin Y, Li Z, Shen J, Chan MT, Wu WK. CCAT1: a pivotal oncogenic long non-coding RNA in human cancers. Cell Prolif. 2016;49:255-260.

10. Bartonicek N, Maag JL, Dinger ME. Long noncoding RNAs in cancer: mechanisms of action and technological advancements. Mol Cancer. 2016;15:43.

11. Gupta RA, Shah N, Wang KC, et al. Long non-coding RNA HOTAIR reprograms chromatin state to promote cancer metastasis. Nature. 2010;464:1071-1076.

12. Yin DD, Liu ZJ, Zhang E, Kong R, Zhang ZH, Guo RH. Decreased expression of long noncoding RNA MEG3 affects cell proliferation and predicts a poor prognosis in patients with colorectal cancer. Tumour Biol. 2015;36:4851-4859.

13. Li Z, Shen J, Chan MT, Wu WK. TUG1: a pivotal oncogenic long noncoding RNA of human cancers. Cell Prolif. 2016;49:471-475.

14. Schmitt AM, Chang HY. Long noncoding RNAs in cancer pathways. Cancer Cell. 2016;29:452-463.

15. Chen WM, Huang MD, Sun DP, et al. Long intergenic non-coding RNA 00152 promotes tumor cell cycle progression by binding to EZH2 and repressing p15 and p21 in gastric cancer. Oncotarget. 2016;7:9773-9787.
16. Wang D, Han S, Peng R, et al. Depletion of histone demethylase KDM5B inhibits cell proliferation of hepatocellular carcinoma by regulation of cell cycle checkpoint proteins p15 and p27. J Exp Clin Cancer Res. 2016;35:37.

17. Xu TP, Liu XX, Xia R, et al. SP1-induced upregulation of the long noncoding RNA TINCR regulates cell proliferation and apoptosis by affecting KLF2 mRNA stability in gastric cancer. Oncogene. 2015;34:5648-5661.

18. Shi Y, Liu Y, Wang J, et al. Downregulated Long Noncoding RNA BANCR Promotes the Proliferation of Colorectal Cancer Cells via Downregualtion of p21 Expression. PLoS ONE. 2015;10:e122679.

19. Kitagawa M, Kitagawa K, Kotake Y, Niida H, Ohhata T. Cell cycle regulation by long non-coding RNAs. Cell Mol Life Sci. 2013;70:4785-4794.

20. Ye LC, Chen T, Zhu DX, et al. Downregulated long non-coding RNA CLMAT3 promotes the proliferation of colorectal cancer cells by targeting regulators of the cell cycle pathway. Oncotarget. 2016; doi: 10.18632/oncotarget.10431. [Epub ahead of print], PMID: 27391344.

21. Lian $\mathrm{Y}$, Ding J, Zhang Z, et al. The long noncoding RNA HOXA transcript at the distal tip promotes colorectal cancer growth partially via silencing of p21 expression. Tumour Biol. 2016;37: 7431-7440.

22. Diederichs S, Bartsch L, Berkmann JC, et al. The dark matter of the cancer genome: aberrations in regulatory elements, untranslated regions, splice sites, non-coding RNA and synonymous mutations. EMBO Mol Med. 2016;8:442-457.

23. Bonasio R, Shiekhattar R. Regulation of transcription by long noncoding RNAs. Annu Rev Genet. 2014;48:433-455.

24. Schmitz SU, Grote P, Herrmann BG. Mechanisms of long noncoding RNA function in development and disease. Cell Mol Life Sci. 2016;73:2491-2509.

25. Kita Y, Yonemori K, Osako Y, et al. Noncoding RNA and colorectal cancer: its epigenetic role. J Hum Genet. 2016; doi: 10.1038/jhg.2016.66. [Epub ahead of print]. PMID: 27278790.

26. Saus E, Brunet-Vega A, Iraola-Guzman S, Pegueroles C, Gabaldon T, Pericay C. Long Non-Coding RNAs As Potential Novel Prognostic Biomarkers in Colorectal Cancer. Front Genet. 2016;7:54.

27. Xie X, Tang B, Xiao YF, et al. Long non-coding RNAs in colorectal cancer. Oncotarget. 2016;7:5226-5239.

28. Huang G, Wu X, Li S, Xu X, Zhu H, Chen X. The long noncoding RNA CASC2 functions as a competing endogenous RNA by sponging miR18a in colorectal cancer. Sci Rep. 2016;6:26524.

29. Wan L, Kong J, Tang J, et al. HOTAIRM1 as a potential biomarker for diagnosis of colorectal cancer functions the role in the tumour suppressor. J Cell Mol Med. 2016; doi: 10.1111/jcmm.12892. [Epub ahead of print] PMID: 27307307

30. Hanahan D, Weinberg RA. Hallmarks of cancer: the next generation. Cell. 2011;144:646-674.

31. Gutschner T, Diederichs $S$. The hallmarks of cancer: a long non-coding RNA point of view. RNA Biol. 2012;9:703-719. 